\title{
Pestwurz als Migräneprophylaxe - allein wirksam oder in einem komplexen Therapieansatz optimierbar?
}

\author{
Eine Kasuistik
}

Rainer Stange, Kathrin Buchcik, Barbara Schwartz, Andreas Michalsen

\section{Anamnese}

Die 54-jährige Patientin weist eine 35-jährige Anamnese mit Migräne auf. In der Woche vor Aufnahme kam es aufgrund einer Exazerbation mit einem 4 Tage währenden Anfall zu einer 13-tägigen vollstationären Behandlung in einer Spezialabteilung für Naturheilkunde. Zuvor waren etwa 2 Anfälle pro Monat aufgetreten bzw. insgesamt etwa $30 \mathrm{im}$ vergangenen Jahr ( $\bullet$ Abb. 1). Eine Migräne-Prophylaxe bestand zu diesem Zeitpunkt nicht. Die Patientin kupiert die Anfälle mit Sumatriptan, das gut wirkt.

Streng linksseitige Migräneattacken mit Aura, visuellen Sehstörungen und Schwindel, Übelkeit, teilweise Erbrechen sowie Licht- und Geruchempfindlichkeit bestanden seit dem 19. Lebensjahr. Der Schmerzcharakter wechselte von dumpf pochend bis zu scharf schneidend und stechend. Es waren keine Zusammenhänge zur Menstruation erkennbar.

\section{Unkonventionelle Therapieversuche ohne Erfolg}

Anfangs hatte sie zur Schmerzbekämpfung Acetylsalicylsäure sowie Ibuprofen eingesetzt, konnte damit aber nur die Spitze der Attacken kupieren. Die Anfälle dauerten bis 48 Stunden, währenddessen die Patientin nicht arbeitsfähig war und sich in einem verdunkelten Raum halbsitzend aufhielt. Später konnte sie die Anfälle mit Sumatriptan besser kupieren. In ihrer langjährigen Anamnese hatte die frühere Zahnarzthelferin und jetzige Heilpraktikerin bereits eine Reihe von unkonventionellen Therapien unternommen, darunter Akupunktur, Rolfing, Shiatsu, Ernährungsumstellung, Homöopathie, Familienaufstellung sowie mikrobiologische Therapie, die jeweils allenfalls minimale Verbesserungen erbracht hatten und nicht über einen längeren Zeitraum eingesetzt wurden.

\section{Erster stationärer Aufenthalt}

Die Patientin hatte bereits knapp 13 Monate zuvor eine damals aus verschiedenen Gründen auf 8 Tage beschränkte vollstationäre Behandlung in derselben Klinik erfahren, in deren Vordergrund jedoch die Komorbiditäten Fibromyalgie-Syndrom (FMS), Infektanfälligkeit bis hin zu einer zuvor durchstandenen Pneumonie sowie ein chronisch rezidivierendes Ekzem unklaren Typs gestanden hatten. Ein unmittelbar zuvor angefertigtes MRT des Kopfes hatte einen unauffälligen Befund erbracht. Sie konnte damals über 4 Tage eine Schleimfastentherapie durchführen. Insbesondere für das FMS hatte sie 3-mal eine wassergefilterte Infrarot-Hyperthermie (wIRA, moderate Therapieführung mit dem Ziel der Erhöhung der Körperkerntemperatur auf maximal $38^{\circ} \mathrm{C}$ ) erhalten und für etwa 6 Monate profitiert. Anamnestisch hatten sich die Beschwerden des FMS durch Wärme, z.B. heiße Bäder und Sauna sowie leichte Bewegung und Spaziergänge bessern lassen; das Ekzem hatte sich durch warmes Wasser jedoch verschlechtert. Infolge der Hyperthermie ließ sich keine Verschlechterung beobachten. Allerdings hatte die Patientin allein während dieses Aufenthaltes 2 Migräne-Anfallstage ohne direkten zeitlichen Zusammenhang etwa zur Hyperthermie erfahren.

\section{Pestwurz-Präparat zur Prophylaxe}

Zum Abschluss war eine Prophylaxe mit einem qualitätsgesicherten PestwurzPräparat (Petadolex ${ }^{\circledR} 50$ mg Kps., Weber und Weber GmbH \& Co. KG, Deutschland) empfohlen und 2 Wochen poststationär auch aufgenommen worden. Die Einnahme begann mit der Dosierung $3 \times 50 \mathrm{mg} / \mathrm{d}$. In den poststationären Monaten 2 und 3 senkte die Patientin die Dosierung auf $2 \times 50 \mathrm{mg} / \mathrm{d}$, da die Migräneanfälle bereits deutlich zurückgegangen waren, in den Monaten 4-7 dann erneut auf $1 \times 50 \mathrm{mg} / \mathrm{d}$, da die Prophylaxe noch bessere Wirkung zeigte und lediglich 3 Anfälle in 6 Monaten erfolgt waren. Es kam dann relativ rasch erneut zu Anfällen ( $\mathbf{A}$ bb. 2 ), sodass die Patientin die Prophylaxe wieder aufnahm. Während der Monate 8-10 setzte die Patientin die Prophylaxe ganz ab ( Abb. 2).

In deren Verlauf war die Frequenz wieder auf die frühere Häufigkeit von 2 Anfällen pro Monat angestiegen, sodass sich im 2. Halbjahr 2015 die Anzahl der Migräneanfälle auf 10 summierte ( $\triangleright$ Abb. 2).

\section{Therapie und Verlauf}

Auch aufgrund des nicht völlig befriedigenden Verlaufes der Migräne-Erkrankung wurde die zweite vollstationäre Behandlung von Beginn an als sog. naturheilkundliche Komplextherapie geplant, die formal über 13 Tage gemäß den Kriterien des OPS-Kataloges durchgeführt wurde (hier OPS 8-975.23). Die Patientin wurde insbesondere motiviert, eine längere Fastentherapie durchzuführen, was nach typischen einleitenden Maßnahmen komplikationslos als Saftfasten nach Buchinger (Leitlinien) über 11 Tage erfolgte. Außerdem erhielt sie 3-mal wöchentlich eine Eigenblutbehandlung mit UVB-Bestrahlung des Blutes (sog. UVBMethode, Gerät Fa. Kastner, Deutschland, Standarddurchführung zweimal 60s) und eine intravenöse Vitamin-C-Therapie mit 6-maliger Gabe von je 7,5g Vitamin C. Hierdurch sollte Erschöpfung, Infektan- 


\begin{tabular}{|c|c|c|c|c|c|c|c|c|c|c|c|}
\hline Januar & Februar & März & April & Mai & Juni & Juli & August & September & Oktober & November & Dezember \\
\hline $1 \mathrm{Mi}$ & $1 \mathrm{Sa}$ & $1 \mathrm{Sa}$ & I Di Migränd & 1 Do & I So Migränd & $1 \mathrm{Di}$ & $1 \mathrm{Fr}$ & $1 \mathrm{Mo}$ & $1 \mathrm{Mi}$ & $1 \mathrm{Sa}$ & $1 \mathrm{Mo}$ \\
\hline 2 Do & 2 Sonntag & 2 Sonntag & $2 \mathrm{Mi}$ & $2 \mathrm{Fr}$ & $2 \mathrm{Mo}$ & $2 \mathrm{Mi}$ & $2 \mathrm{Sa}$ & $2 \mathrm{Di}$ & 2 Do & 2 Sonntag & 12Di Migräne \\
\hline $3 \mathrm{Fr}$ & $3 \mathrm{Mo}$ & $3 \mathrm{Mo}$ & 3 Do & $3 \mathrm{Sa}$ & $3 \mathrm{Di}$ & 3 Do & 3 Sonntag & $3 \mathrm{Mi}$ & $3 \mathrm{Fr}$ & $3 \mathrm{Mo}$ & $3 \mathrm{Mi}$ \\
\hline 4Sa Mirräne & $4 \mathrm{Di}$ & $4 \mathrm{Di}$ & $4 \mathrm{Fr}$ & 4 Sonntag & $4 \mathrm{Mi}$ & $4 \mathrm{Fr}$ & $4 \mathrm{Mo}$ & 4 Do & $4 \mathrm{Sa}$ & $4 \mathrm{Di}$ & 4 Do \\
\hline 5 Sonntag & $5 \mathrm{Mi}$ & $5 \mathrm{Mi}$ & $5 \mathrm{Sa}$ & $5 \mathrm{Mo}$ & 5 Do & $5 \mathrm{Sa}$ & $5 \mathrm{Di}$ & $5 \mathrm{Fr}$ & 5 Sonntag & $5 \mathrm{Mi}$ & $5 \mathrm{Fr}$ \\
\hline $6 \mathrm{Mo}$ & 6 Do & 6 Do & 6 Sonntag & $6 \mathrm{Di}$ & $6 \mathrm{Fr}$ & 6 Sonntag & $6 \mathrm{Mi}$ & $6 \mathrm{Sa}$ & $6 \mathrm{Mo}$ & 6. Do Migräne & $6 \mathrm{Sa}$ \\
\hline $7 \mathrm{Di}$ & $7 \mathrm{Fr}$ & $7 \mathrm{Fr}$ & $7 \mathrm{Mo}$ & $7 \mathrm{Mi}$ & $7 \mathrm{Sa}$ & $7 \mathrm{Mo}$ & 7 Do & 7 Sonntag & $7 \mathrm{Di}$ & 7 Fr Mierräne & 7 Sonntag \\
\hline $8 \mathrm{Mi}$ & $8 \mathrm{Sa}$ & $8 \mathrm{Sa}$ & $8 \mathrm{Di}$ & 8 Do & 8 Sonntag & 8. Di Migräni & $8 \mathrm{Fr}$ & $8 \mathrm{Mo}$ & $8 \mathrm{Mi}$ & $8 \mathrm{Sa}$ & $8 \mathrm{Mo}$ \\
\hline 9 Do & 9 Sonntag & 9 Sonntag & $9 \mathrm{Mi}$ & $9 \mathrm{Fr}$ & 9 Mo & $9 \mathrm{Mi}$ & OSa Migräne & $9 \mathrm{Di}$ & Do Migräne & 9 Sonntag & Q Di Migräne \\
\hline $10 \mathrm{Fr}$ & 10 Mo & $10 \mathrm{Mo}$ & 10 Do & $10 \mathrm{Sa}$ & $10 \mathrm{Di}$ & 10 Do & 10 Sonntag & $10 \mathrm{Mi}$ & L0 Fr Mirgäne & $10 \mathrm{Mo}$ & $10 \mathrm{Mi}$ \\
\hline $11 \mathrm{Sa}$ & $11 \mathrm{Di}$ & $11 \mathrm{Di}$ & $11 \mathrm{Fr}$ & 11 Sonntag & $11 \mathrm{Mi}$ & $11 \mathrm{Fr}$ & $11 \mathrm{Mo}$ & 11 Do & $11 \mathrm{Sa}$ & $11 \mathrm{Di}$ & II DoMieräne \\
\hline \begin{tabular}{|l|}
12 Sonntag \\
\end{tabular} & $12 \mathrm{Mi}$ & $12 \mathrm{Mi}$ & $12 \mathrm{Sa}$ & $12 \mathrm{Mo}$ & 12 Do & $12 \mathrm{Sa}$ & $12 \mathrm{Di}$ & $12 \mathrm{Fr}$ & 12 Sonntag & $12 \mathrm{Mi}$ & 12. FrMioräne \\
\hline $13 \mathrm{Mo}$ & 13 Do Migrän & 13 Do & 13 Sonntag & $13 \mathrm{Di}$ & $13 \mathrm{Fr}$ & 13 Sonntag & $13 \mathrm{Mi}$ & $13 \mathrm{Sa}$ & $13 \mathrm{Mo}$ & 13 Do & $13 \mathrm{Sa}$ \\
\hline $14 \mathrm{Di}$ & $14 \mathrm{Fr}$ & $14 \mathrm{Fr}$ & $14 \mathrm{Mo}$ & $14 \mathrm{Mi}$ & $14 \mathrm{Sa}$ & $14 \mathrm{Mo}$ & 14 Do & 14 Sonntag & $14 \mathrm{Di}$ & $14 \mathrm{Fr}$ & 14 Sonntag \\
\hline $15 \mathrm{Mi}$ & $15 \mathrm{Sa}$ & $15 \mathrm{Sa}$ & $15 \mathrm{Di}$ & 15 Do & 15 Sonntag & $15 \mathrm{Di}$ & $15 \mathrm{Fr}$ & $15 \mathrm{Mo}$ & $15 \mathrm{Mi}$ & $15 \mathrm{Sa}$ & $15 \mathrm{Mo}$ \\
\hline 16 Do & 16 Sonntag & 16 Sonntag & $16 \mathrm{Mi}$ & $16 \mathrm{Fr}$ & $16 \mathrm{Mo}$ & $16 \mathrm{Mi}$ & $16 \mathrm{Sa}$ & $16 \mathrm{Di}$ & 16 Do & 16 So Migräne & 16 DiMigrän \\
\hline $17 \mathrm{Fr}$ & $17 \mathrm{Mo}$ & $17 \mathrm{Mo}$ & 17 Do & $17 \mathrm{Sa}$ & $17 \mathrm{Di}$ & 17 Do Migräne & 17 Sonntag & 17 Mi Migräne & $17 \mathrm{Fr}$ & $17 \mathrm{Mo}$ & 17. MiMigräne \\
\hline $18 \mathrm{Sa}$ & $18 \mathrm{Di}$ & $18 \mathrm{Di}$ & $18 \mathrm{Fr}$ & 18 Sonntag & $18 \mathrm{Mi}$ & 18 Fr Migräne & $18 \mathrm{Mo}$ & 18 Do & $18 \mathrm{Sa}$ & $18 \mathrm{Di}$ & 18 Do \\
\hline 19 Sonntag & $19 \mathrm{Mi}$ & $19 \mathrm{Mi}$ & $19 \mathrm{Sa}$ & 19 Mo & 19 Do & 19 Sa Migränt & $19 \mathrm{Di}$ & $19 \mathrm{Fr}$ & 19 Sonntag & $19 \mathrm{Mi}$ & $19 \mathrm{Fr}$ \\
\hline $20 \mathrm{Mo}$ & 20 Do & 20 Do & 20 Sonntag & $20 \mathrm{Di}$ & $20 \mathrm{Fr}$ & 20 Sonntag & $20 \mathrm{Mi}$ & $20 \mathrm{Sa}$ & $20 \mathrm{Mo}$ & 20 Do Migräne & $20 \mathrm{Sa}$ \\
\hline $21 \mathrm{Di}$ & $21 \mathrm{Fr}$ & $21 \mathrm{Fr}$ & $21 \mathrm{Mo}$ & $21 \mathrm{Mi}$ & $21 \mathrm{Sa}$ & $21 \mathrm{Mo}$ & 21 Do & 21 Sonntag & $21 \mathrm{Di}$ & $21 \mathrm{Fr}$ & 21 Sonntag \\
\hline $22 \mathrm{Mi}$ & $22 \mathrm{Sa}$ & $22 \mathrm{Sa}$ & 22 Di & 22 Do & 22 Sonntag & $22 \mathrm{Di}$ & $22 \mathrm{Fr}$ & $22 \mathrm{Mo}$ & $22 \mathrm{Mi}$ & $22 \mathrm{Sa}$ & $22 \mathrm{Mo}$ \\
\hline 23 Do & 23 So Migräne & 23 Sonntag & $23 \mathrm{Mi}$ & $23 \mathrm{Fr}$ & $23 \mathrm{Mo}$ & $23 \mathrm{Mi}$ & $23 \mathrm{Sa}$ & $23 \mathrm{Di}$ & 23 Do & 23 Sonntag & $23 \mathrm{Di}$ \\
\hline $24 \mathrm{Fr}$ & $24 \mathrm{Mo}$ & $24 \mathrm{Mo}$ & 24 Do & $24 \mathrm{Sa}$ & $24 \mathrm{Di}$ & 24 Do & 24 Sonntag & $24 \mathrm{Mi}$ & $24 \mathrm{Fr}$ & $24 \mathrm{Mo}$ & $24 \mathrm{Mi}$ \\
\hline $25 \mathrm{Sa}$ & $25 \mathrm{Di}$ & $25 \mathrm{Di}$ & $25 \mathrm{Fr}$ & 25 Sonntag & $25 \mathrm{Mi}$ & $25 \mathrm{Fr}$ & $25 \mathrm{Mo}$ & 25 Do & $25 \mathrm{Sa}$ & 25 DiMigräne & 25 Do \\
\hline 26 Sonntag & $26 \mathrm{Mi}$ & $26 \mathrm{Mi}$ & $26 \mathrm{Sa}$ & $26 \mathrm{Mo}$ & 26 Do & $26 \mathrm{Sa}$ & $26 \mathrm{Di}$ & $26 \mathrm{Fr}$ & 26 Sonntag & $26 \mathrm{Mi}$ & $26 \mathrm{Fr}$ \\
\hline $27 \mathrm{Mo}$ & 27 Do Migräne & 27 Do & 27 Sonntag & 27 Di Migräne & $27 \mathrm{Fr}$ & 27 Sonntag & $27 \mathrm{Mi}$ & $27 \mathrm{Sa}$ & $27 \mathrm{Mo}$ & 27 Do & $27 \mathrm{Sa}$ \\
\hline $28 \mathrm{Di}$ & $28 \mathrm{Fr}$ & $28 \mathrm{Fr}$ & $28 \mathrm{Mo}$ & $28 \mathrm{Mi}$ & $28 \mathrm{Sa}$ & $28 \mathrm{Mo}$ & 28 Do & 28 Sonntag & $28 \mathrm{Di}$ & $28 \mathrm{Fr}$ & 28 Sonntag \\
\hline 29 Mi Miøräne & & $29 \mathrm{Sa}$ & $29 \mathrm{Di}$ & 29 Do & 29 Sonntag & $29 \mathrm{Di}$ & $29 \mathrm{Fr}$ & $29 \mathrm{Mo}$ & 29 Mireäne & $29 \mathrm{Sa}$ & $29 \mathrm{Mo}$ \\
\hline 30 Do & & 30 Sonntag & $30 \mathrm{Mi}$ & $30 \mathrm{Fr}$ & $30 \mathrm{Mo}$ & B0 Mi Migräne & $30 \mathrm{Sa}$ & $30 \mathrm{Di}$ & B0 Mieräne & 30 Sonntag & $30 \mathrm{Di}$ \\
\hline $31 \mathrm{Fr}$ & & $31 \mathrm{Mo}$ & & 31.Sa & & 31 Do & 31 Sonntag & & $31 \mathrm{Fr}$ & & $31 \mathrm{Mi}$ \\
\hline
\end{tabular}

\begin{tabular}{|c|c|c|c|c|c|c|c|c|c|c|c|}
\hline \begin{tabular}{|l|} 
Januar \\
\end{tabular} & Februar & März & April & Mai & Juni & Juli & August & September & Oktober & November & Dezember \\
\hline 1 Sa Petadolex & 1 So & 1 Sonntag & $1 \mathrm{Mi}$ & $1 \mathrm{Fr}$ & $1 \mathrm{Mo}$ & $1 \mathrm{Mi}$ & $1 \mathrm{Sa}$ & $1 \mathrm{Di}$ & 1 Do & 1 Sonntag & $1 \mathrm{Di}$ \\
\hline $2 \mathrm{Fr}$ & $2 \mathrm{Mo}$ & $2 \mathrm{Mo}$ & 2 Do & $2 \mathrm{Sa}$ & $2 \mathrm{Di}$ & 2 Do & 2 Sonntag & $2 \mathrm{Mi}$ & $2 \mathrm{Fr}$ & $2 \mathrm{Mo}$ & $2 \mathrm{Mi}$ \\
\hline $3 \mathrm{Sa}$ & $3 \mathrm{Di}$ & $3 \mathrm{Di}$ & $3 \mathrm{Fr}$ & 3 Sonntag & $3 \mathrm{Mi}$ & $3 \mathrm{Fr}$ & $3 \mathrm{Mo}$ & 3 Do & $3 \mathrm{Sa}$ & 3. Di Mimäne & 3 Do \\
\hline 4 Sonntag & $4 \mathrm{Mi}$ & $4 \mathrm{Mi}$ & $4 \mathrm{Sa}$ & $4 \mathrm{Mo}$ & 4 Do & $4 \mathrm{Sa}$ & $4 \mathrm{Di}$ & $4 \mathrm{Fr}$ & 4 Sonntag & 4 Mi Petadolex & $4 \mathrm{Fr}$ \\
\hline $5 \mathrm{Mo}$ & 5 Do & 5 Do & 5 Sonntag & $5 \mathrm{Di}$ & $5 \mathrm{Fr}$ & 5 Sonntag & $5 \mathrm{Mi}$ & $5 \mathrm{Sa}$ & $5 \mathrm{Mo}$ & 5 Do & $5 \mathrm{Sa}$ \\
\hline $6 \mathrm{Di}$ & $6 \mathrm{Fr}$ & $6 \mathrm{Fr}$ & $6 \mathrm{Mo}$ & $6 \mathrm{Mi}$ & 6. Sa Migräne & $6 \mathrm{Mo}$ & 6 Do & 6 Sonntag & $6 \mathrm{Di}$ & $6 \mathrm{Fr}$ & 6 Sonntag \\
\hline $7 \mathrm{Mi}$ & $7 \mathrm{Sa}$ & $7 \mathrm{Sa}$ & $7 \mathrm{Di}$ & 7 Do & 7 Sonntag & $7 \mathrm{Di}$ & $7 \mathrm{Fr}$ & $7 \mathrm{Mo}$ & $7 \mathrm{Mi}$ & 7 Sa Mipräne & $7 \mathrm{Mo}$ \\
\hline \begin{tabular}{|l|} 
8 Do \\
\end{tabular} & 8 Sonntag & 8 Sonntag & 8 Mi Migräne & $8 \mathrm{Fr}$ & $8 \mathrm{Mo}$ & $8 \mathrm{Mi}$ & $8 \mathrm{Sa}$ & $8 \mathrm{Di}$ & 8 Do & 8 Sonntag & $8 \mathrm{Di}$ \\
\hline $9 \mathrm{Fr}$ & 9 Mo & $9 \mathrm{Mo}$ & 9 Do & $9 \mathrm{Sa}$ & $9 \mathrm{Di}$ & 9 Do & 9 Sonntag & 2. Mi Mirräne & $9 \mathrm{Fr}$ & 9 Mo & $9 \mathrm{Mi}$ \\
\hline $10 \mathrm{Sa}$ & $10 \mathrm{Di}$ & $10 \mathrm{Di}$ & $10 \mathrm{Fr}$ & 10 Sonntag & $10 \mathrm{Mi}$ & $10 \mathrm{Fr}$ & $10 \mathrm{Mo}$ & $10 \mathrm{Do}$ & $10 \mathrm{Sa}$ & $10 \mathrm{Di}$ & $10 \mathrm{Do}$ \\
\hline 11 Sonntag & $11 \mathrm{Mi}$ & $11 \mathrm{Mi}$ & $11 \mathrm{Sa}$ & $11 \mathrm{Mo}$ & 11 Do & $11 \mathrm{Sa}$ & $11 \mathrm{Di}$ & $11 \mathrm{Fr}$ & 11 Sonntag & $11 \mathrm{Mi}$ & $11 \mathrm{Fr}$ \\
\hline $12 \mathrm{Mo}$ & 12 Do & 12 Do & \begin{tabular}{|l}
12 Sonntag \\
\end{tabular} & $12 \mathrm{Di}$ & $12 \mathrm{Fr}$ & \begin{tabular}{|l}
12 Sonntag \\
\end{tabular} & $12 \mathrm{Mi}$ & $12 \mathrm{Sa}$ & $12 \mathrm{Mo}$ & 12 Do & $12 \mathrm{Sa}$ \\
\hline $13 \mathrm{Di}$ & $13 \mathrm{Fr}$ & $13 \mathrm{Fr}$ & $13 \mathrm{Mo}$ & $13 \mathrm{Mi}$ & $13 \mathrm{Sa}$ & $13 \mathrm{Mo}$ & 13 Do & 13 Sonntag & $13 \mathrm{Di}$ & $13 \mathrm{Fr}$ & 13 Sonntag \\
\hline $14 \mathrm{Mi}$ & $14 \mathrm{Sa}$ & $14 \mathrm{Sa}$ & $14 \mathrm{Di}$ & 14 Do & 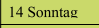 & $14 \mathrm{Di}$ & $14 \mathrm{Fr}$ & $14 \mathrm{Mo}$ & $14 \mathrm{Mi}$ & $14 \mathrm{Sa}$ & $14 \mathrm{Mo}$ \\
\hline 15 Do & I5 So Migräne & 15 Sonntag & $15 \mathrm{Mi}$ & $15 \mathrm{Fr}$ & $15 \mathrm{Mo}$ & $15 \mathrm{Mi}$ & $15 \mathrm{Sa}$ & $15 \mathrm{Di}$ & 15 Do & 15 Sonntag & $15 \mathrm{Di}$ \\
\hline $16 \mathrm{Fr}$ & $16 \mathrm{Mo}$ & $16 \mathrm{Mo}$ & 16 Do & $16 \mathrm{Sa}$ & $16 \mathrm{Di}$ & 16 Do & 16 Sonntag & $16 \mathrm{Mi}$ & $16 \mathrm{Fr}$ & $16 \mathrm{Mo}$ & $16 \mathrm{Mi}$ \\
\hline $17 \mathrm{Sa}$ & $17 \mathrm{Di}$ & $17 \mathrm{Di}$ & $17 \mathrm{Fr}$ & 17 Sonntag & $17 \mathrm{Mi}$ & $17 \mathrm{Fr}$ & $17 \mathrm{Mo}$ & 17 Do & $17 \mathrm{Sa}$ & $17 \mathrm{Di}$ & 17 Do \\
\hline 18 Sonntag & $18 \mathrm{Mi}$ & $18 \mathrm{Mi}$ & $18 \mathrm{Sa}$ & $18 \mathrm{Mo}$ & 18 Do & $18 \mathrm{Sa}$ & $18 \mathrm{Di}$ & $18 \mathrm{Fr}$ & 18 So Migräne & $18 \mathrm{Mi}$ & $18 \mathrm{Fr}$ \\
\hline $19 \mathrm{Mo}$ & 19 Do & 19 Do & 19 Sonntag & $19 \mathrm{Di}$ & $19 \mathrm{Fr}$ & 19 Sonntag & 19 Mi Mierăne & $19 \mathrm{Sa}$ & $19 \mathrm{Mo}$ & 19 Do & $19 \mathrm{Sa}$ \\
\hline $20 \mathrm{Di}$ & $20 \mathrm{Fr}$ & $20 \mathrm{Fr}$ & 20 Mo & $20 \mathrm{Mi}$ & $20 \mathrm{Sa}$ & $20 \mathrm{Mo}$ & $20 \mathrm{Do}$ & 20 Sonntag & $20 \mathrm{Di}$ & $20 \mathrm{Fr}$ & 20 Sonntag \\
\hline $21 \mathrm{Mi}$ & $21 \mathrm{Sa}$ & $21 \mathrm{Sa}$ & $21 \mathrm{Di}$ & 21 Do & 21 Sonntag & $21 \mathrm{Di}$ & $21 \mathrm{Fr}$ & $21 \mathrm{Mo}$ & $21 \mathrm{Mi}$ & $21 \mathrm{Sa}$ & $21 \mathrm{Mo}$ \\
\hline 22 Do & 22 Sonntag & 22 Sonntag & $22 \mathrm{Mi}$ & $22 \mathrm{Fr}$ & $22 \mathrm{Mo}$ & $22 \mathrm{Mi}$ & $22 \mathrm{Sa}$ & 22 Di Mirzäne & 22 Do & 22 Sonntag & $22 \mathrm{Di}$ \\
\hline $23 \mathrm{Fr}$ & $23 \mathrm{Mo}$ & $23 \mathrm{Mo}$ & 23 Do & $23 \mathrm{Sa}$ & $23 \mathrm{Di}$ & 23 Do & 23 Sonntag & $23 \mathrm{Mi}$ & $23 \mathrm{Fr}$ & $23 \mathrm{Mo}$ & $23 \mathrm{Mi}$ \\
\hline $24 \mathrm{Sa}$ & $24 \mathrm{Di}$ & $24 \mathrm{Di}$ & $24 \mathrm{Fr}$ & 24 Sonntag & $24 \mathrm{Mi}$ & $24 \mathrm{Fr}$ & $24 \mathrm{Mo}$ & 24 Do & $24 \mathrm{Sa}$ & 24 Di Migräne & 24 Do \\
\hline 25 Sonntag & $25 \mathrm{Mi}$ & $25 \mathrm{Mi}$ & $25 \mathrm{Sa}$ & $25 \mathrm{Mo}$ & 25 Do & 25. Sa Miprăne & 25 Do & $25 \mathrm{Fr}$ & 25 Sonntag & $25 \mathrm{Mi}$ & $25 \mathrm{Fr}$ \\
\hline $26 \mathrm{Mo}$ & 26 Do & 26 Do & 26 Sonntag & $26 \mathrm{Di}$ & $26 \mathrm{Fr}$ & 26 Sonntag & $26 \mathrm{Mi}$ & $26 \mathrm{Ss}$ & $26 \mathrm{Mo}$ & 26 Do & $26 \mathrm{Ss}$ \\
\hline $27 \mathrm{Di}$ & $27 \mathrm{Fr}$ & $27 \mathrm{Fr}$ & $27 \mathrm{Mo}$ & $27 \mathrm{Mi}$ & $27 \mathrm{Sa}$ & $27 \mathrm{Mo}$ & 27 Do & 27 Sonntag & $27 \mathrm{Di}$ & $27 \mathrm{Fr}$ & 27 Sonntag \\
\hline $28 \mathrm{Mi}$ & $28 \mathrm{Sa}$ & $28 \mathrm{Sa}$ & $28 \mathrm{Di}$ & 28 Do & 28 Sonntag & $28 \mathrm{Di}$ & $28 \mathrm{Fr}$ & $28 \mathrm{Mo}$ & $28 \mathrm{Mi}$ & $28 \mathrm{Sa}$ & $28 \mathrm{Mo}$ \\
\hline 29 Do & & 29 Sonntag & $29 \mathrm{Mi}$ & $29 \mathrm{Fr}$ & $29 \mathrm{Mo}$ & $29 \mathrm{Mi}$ & 29 Sa Mieräne & $29 \mathrm{Di}$ & 29 Do Migräne & 29 Sonntag & $29 \mathrm{Di}$ \\
\hline $30 \mathrm{Fr}$ & & $30 \mathrm{Mo}$ & 30 Do & $30 \mathrm{Sa}$ & $30 \mathrm{Di}$ & 30 Do kein & 30 So & $30 \mathrm{Mi}$ & $30 \mathrm{Fr}$ & $30 \mathrm{Mo}$ & $30 \mathrm{Mi}$ \\
\hline $31 \mathrm{Sa}$ & & 31 Di & & 31 So & & 31 FrPetadolex & 1 Mo & & $31 \mathrm{Sa}$ & & 31 Do \\
\hline
\end{tabular}

Petadolex (Pestwurz) verordnet von Dr. Stange - Naturheilkundliche Abteilung Immanuel Krankenhaus im Dezember 2014

Einnahme 2015:

Januar

Februar / März

April / Mai / Juni / Juli

August / September / Oktober

November

Dezember 3x1 Kapsel

2x1 Kapsel

1x1 Kapsel

PAUSE, keine Kapseln

3x1 Kapsel

2x1 Kapsel
- Abb. 1 Kalenderjahr 2014: 32 Migräneanfälle.
- Abb. 2 Kalenderjahr 2015: 13 Migräneanfälle. An grün unterlegten Tagen wurde eine Prophylaxe mit Pestwurz eingenommen. 
fälligkeit und das eventuell allergische Exanthem durch Umstimmung des Immunsystems positiv beeinflusst werden. Daneben erfolgte eine naturheilkundliche multimodale Komplexbehandlung mit Hyperthermie-Serie, Atemtherapie-Gruppe, reflektorischer Atemtherapie in Einzelsitzungen, Frühsport, Hockergymnastik, Wasserlaufgruppe, progressiver Muskelrelaxation nach Jacobsen sowie motorischfunktioneller Behandlung der Schultergelenke.

Nach diesem weiteren Aufenthalt im November 2015 wurde die Pestwurz-Prophylaxe erneut mit $3 \times 50 \mathrm{mg} / \mathrm{d}$ aufgenommen. In den 6 Monaten seitdem ist kein weiterer Anfall erfolgt.

\section{Diskussion}

Die Kasuistik weist auf eine dosisabhängige Wirksamkeit einer Pestwurz-Prophylaxe bei Migräne hin. Dies zeigt sich auch anhand des sehr sorgfältig geführten Anfallstagebuchs. Unterstützt wird diese Annahme durch das Anfallsrezidiv nach völligem Absetzen und die erneute Remission bei Wiederaufnahme der Prophylaxemedikation.

Pestwurz-Extrakte weisen entzündungshemmende und spasmolytische Wirkungen auf, deretwegen sie seit geraumer Zeit genutzt und auch zunehmend wissenschaftlich beforscht werden [1-5].

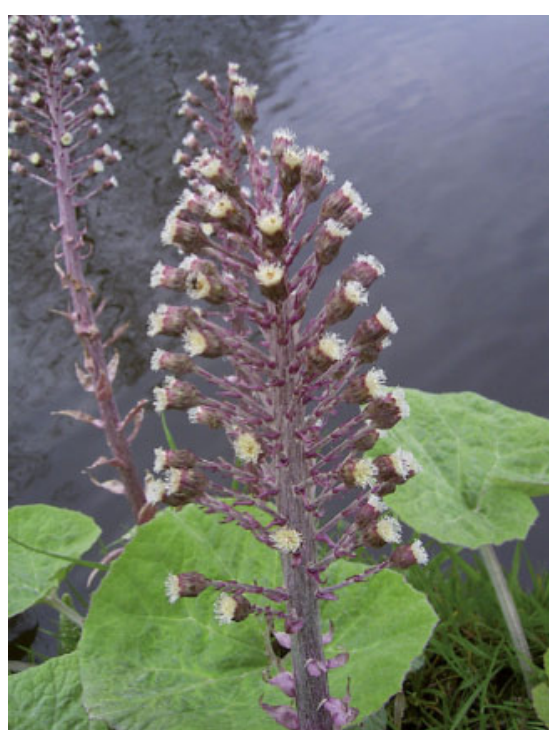

- Abb. 3 Pestwurz (Petasites hybridus). (C) Teun Spaans
Sie scheinen grundsätzlich als MigräneProphylaktikum geeignet, wenngleich die lange verfolgte Theorie meningealer Gefäßspasmen als Ursache der Migräneattacken in letzter Zeit erheblich in Zweifel gezogen wurde.

Neben Behandlung und Prophylaxe von allergischen und bronchialobstruktiven Erkrankungen [6] werden Pestwurz-Extrakte seit geraumer Zeit ausschließlich für die Prophylaxe der Migräne empfohlen; eine therapeutische Wirkung im Anfall ist dagegen nicht beschrieben.

Es liegen mindestens 4 randomisiert placebokontrollierte Studien und mindestens 2 Reviews vor: Der erste [7] überblickt 2 als hochwertig eingestufte Studien mit 60 bzw. 233 Patienten [8, 9], wobei in der größeren Studie 2 Verumgruppen mit 100 und $150 \mathrm{mg} / \mathrm{d}$ gebildet worden waren. Der Review kommt zu dem Schluss, dass nach 3-4 Monaten in beiden Verumgruppen mit der Dosis von $150 \mathrm{mg} / \mathrm{d}$ die Anfallsfrequenz deutlicher sank und die Responderraten höher waren als mit $100 \mathrm{mg} / \mathrm{d}$. Ein Poolen der Daten wurde aus verschiedenen Gründen nicht für sinnvoll erachtet, es müssten mehr und methodologisch bessere Studien vorliegen.

Obwohl nahezu zeitgleich publiziert, würdigt der andere Review [10] 2 weitere randomisiert placebokontrollierte Studien $[11,12]$, insgesamt also 4 , die sämtlich nach ca. 4 Monaten auf eine bessere Wirksamkeit des Verums gegenüber Placebo hinwiesen. Die gewählten Dosierungen variierten zwischen $2 \times 25 \mathrm{mg} / \mathrm{d}$ und $3 \times 50 \mathrm{mg} / \mathrm{d}$; bessere Ergebnisse erzielten die höheren Dosierungen. Es wird insgesamt ein Evidenzgrad B verliehen (Good Scientific Evidence, Skala von A [maximal] bis E [minimal]).

\section{Sicherheit}

Pestwurz-Extrakte gelten als gut verträglich. Sie werden in Deutschland seit 1972, in Kanada seit 1996, in den USA spätestens seit 1998 und in Japan seit 2003 in größerem Umfang als Migräne-, in jüngerer Vergangenheit aber auch vermehrt als Allergieprophylaxe eingesetzt. Aus Deutschland wurden 9 Verdachtsfälle bezüglich Leberschäden gemeldet und sorgfältig analysiert. In einem Fall schien ein Zusammenhang möglich [13]. Bezüglich des grundsätzlich möglichen Gehaltes an als krebserregend und lebertoxisch eingestuften Pyrrolizidinalkaloiden ließ das National Center for Natural Products Research 21 in den USA erhältliche Pestwurz-Extrakte analysieren und befand sie für sicher. Andere ernsthafte unerwünschte Wirkungen wurden weder in den Studien noch aus der breiten Anwendung beschrieben.

Für das Produkt Petadolex ${ }^{\circledR}$ lässt sich in Deutschland der dauerhafte Gebrauch zwischen 1992 und 2010 während etwa 200000 Patientenjahren überschauen, für die USA, Kanada und Japan zusammen zwischen 2010 und 2015 während etwa 56000 Patientenjahren.

\section{Erhältlichkeit und Kosten}

Auf einem Methylenchlorid-Extraktionsverfahren beruhende Pestwurz-Arzneimittel waren in Deutschland seit 1972 erhältlich. Der Hersteller verlor nach der 1988 erfolgten Umstellung auf ein moderneres Kohlendioxid-Extraktionsverfahren im Dissens mit dem Bundesinstitut für Arzneimittel und Medizinprodukte ab 2009 die Vermarktungsgenehmigung für Deutschland. Nur der letztgenannte Extrakt wurde in den Migränestudien getestet. Auf diesem Extrakt gründende Arzneimittel werden unverändert in Deutschland produziert, sind aber nur über eine internationale Bezugsquelle erhältlich.

Zwischen 1992 und 2004 war darüber hinaus als offenbar weltweit einziger qualitätsgesicherter Extrakt ein Schweizer Produkt (Petaforce ${ }^{\circledR}$, Fa. Zeller, Schweiz) erhältlich. Dessen Hersteller beansprucht aufgrund eines anderen Verhältnisses der grundsätzlich als Stereoisomere vorliegenden und allgemein als wirksamkeitsbestimmend anerkannten Petasine eine bessere Wirksamkeit bei Allergien [14, 15]. Auch hierzu liegen mehrere doppelblinde, randomisierte Studien im Vergleich zu Placebo und - im Unterschied zur Indikation Migräne - auch zum Standardtherapeutikum Ceterizin vor. Dieser Extrakt ist bislang bei Migräne in keiner randomisierten Studie untersucht worden, ebenso wenig wie der andere bei Allergien. Dort wurde der Aufsichtsbehörde Swissmedic 
ein Verdachtsfall aus Deutschland bezüglich Lebertoxizität angezeigt.

Die Kosten belaufen sich je nach Bestellmenge auf $0,66-0,52 €$ pro $50 \mathrm{mg}-$ Kapsel. In der derzeit empfohlenen Dosierung von $3 \times 50 \mathrm{mg} / \mathrm{d}$ entstehen somit Tagestherapiekosten von ca. 1,80€. Diese werden von der gesetzlichen Krankenversicherung grundsätzlich nicht übernommen. Ob eine partielle Rückvergütung im Rahmen der von vielen GKV-Partnern angebotenen pauschalen Bezuschussungsmodelle nach ärztlicher Verordnung über ein privates bzw. Grünes Rezept erfolgen kann, ist eine Kulanzfrage, da es sich bei beiden Pestwurz-Produkten zwar um rezeptfreie, aber nicht in Deutschland zugelassene Arzneimittel handelt. In der privaten Krankenversicherung und den Beihilfen dürften Erstattungsgepflogenheiten unterschiedlich ausfallen. In jedem Fall erscheint hier bei unterschiedlichen Auffassungen ein Hinweis auf die Literaturlage und den individuellen Prophylaxe-Erfolg nützlich, insbesondere angesichts hoher, jedoch von keinem Versicherer limitierter Kosten für Triptane. Liegen hierzu für den Einzelfall genügend Daten vor, erscheint auch die Beantragung einer dauerhaften Ausnahmeregelung aussichtsreich.

\section{Fazit}

Im vorliegenden Fall scheint eine reproduzierbare prophylaktische Wirkung eines standardisierten Pestwurz-Extraktes bei einer ausgeprägten Migräneerkrankung vorzuliegen. Unklar bleibt ein möglicher additiver Beitrag von Therapien, die in den beiden vollstationären Verfahren in einer naturheilkundlichen Spezialklinik ebenfalls eingesetzt, jedoch in der ambulanten Situation nicht weiter fortgeführt wurden. Hier ist z. B. an die UVBBehandlung der Patientin zu erinnern (s.o.), die grundsätzlich bei vaskulären Erkrankungen eines ihrer Hauptindikationsgebiete hat. Migräne ist bislang von ihren Vertretern allerdings nicht hervorgehoben worden.

In erster Linie ist jedoch die 11-tägige Fastentherapie zu nennen, da nach der zweiten vollstationären Therapie die Pro- phylaxe bislang offenbar erfolgreicher war. Therapeutisches Fasten wird seit geraumer Zeit von erfahrenen Fastenärzten als alleinige Maßnahme bei Migräne empfohlen, die weltweit einzige ärztliche Fachgesellschaft gibt diese Indikation in ihren älteren deutsch- wie jüngeren englischsprachigen Leitlinien aus [16, 17]. Wissenschaftliche Untersuchungen sind bislang allerdings nur in Form einer schon älteren Dissertation bekannt geworden, die retrospektiv eine Auswertung von Langzeitverläufen von Patienten einer großen Fastenklinik beinhaltet (Brückenau). Der Wirkmechanismus ist unbekannt, könnte jedoch auf den im Modell chronisch entzündlicher Gelenkerkrankungen gesicherten entzündungshemmenden Wirkungen sowie der auch in anderen Zusammenhängen beobachteten vegetativ stabilisierenden Wirkung des Fastens beruhen. Völlig unbekannt, wenngleich grundsätzlich zu unterstellen, ist, ob es zu einem kombinierten Effekt der beiden Therapien kommt.

Interessenkonflikt: Alle Autoren erklären, dass sie im Zusammenhang mit dem Gegenstand des Artikels keine Interessenkonflikte haben.

Dr. med. Rainer Stange

Immanuel Krankenhaus

Königstr. 63

14109 Berlin

r.stange@immanuel.de

Online

http://dx.doi.org/10.1055/s-0042-109894

\section{Literatur}

1 Aebi A, Buechi J, Waaler T et al. Inhaltsstoffe von Petasites hybridus (L) FI Wett I. Pharm Acta Helv 1955; 29: 277-279

2 Bucher K. Über ein antispastisches Prinzip in Petasites officinalis Moench. Arch Exp Pathol Pharmakol 1951; 213: 69-71

3 Brune K, Bickel D, Peskar BA. Gastro-protective effects by extracts of Petasites hybridus. The role of inhibition of peptido-leukotriene synthesis. Planta Med 1993; 59: 494-496

4 Scheidegger C, Dahinden C, Wiesmann U. Effects of extracts of individual components from Petasites on prostaglandin synthesis in cultured skin fibroblasts and on leucotriene synthesis in isolated human peripheral leucocytes. Pharm Acta Helv 1998; 72 : 359-380

5 Thomet OA, Wiesmann UN, Schapowal A et al. Role of petasin in the potential anti-inflammatory activity of a plant extract of Petasites hybridus. Biochem Pharmacol 2001; 61: 1041-1047

6 Ziolo G, Samochewiec L. Study on clinical properties and mechanism of action of Petasites in bronchial asthma and chronic obstructive bronchitis. Pharm Acta Helv 1998; 72: 359-380

7 Agosti R, Duke RK, Chrubasik JE, Chrubasik S. Effectiveness of Petasites hybridus preparations in the prophylaxis of migraine: a systematic review. Phytomedicine 2006; 13: 743-746

8 Diener HC, Rahlfs VW, Danesch U. The first placebo-controlled trial of a special butterbur root extract for the prevention of migraine: reanalysis of efficacy criteria. Eur Neurol 2004; 51: 89-97

9 Lipton RB, Göbel H, Einhäupl KM et al. Petasites hybridus root (butterbur) is an effective preventive treatment for migraine. Neurology 2004; 63: 2240-2244

10 Giles M, Ulbricht C, Khalsa KP et al. Butterbur. An evidence-based systematic review by the Natural Standard Research Collaboration. J Herbal Pharmacotherapy 2005; 5: 119-143

11 Grossmann M, Schmidramsl H. An extract of Petasites hybridus is effective in the prophylaxis of migraine. Int J Clin Pharmacol Ther 2000; 38: 430-435

12 Degenring FH, Bommer S. Prévention de la migraine par Petadolor $\mathrm{H}$ (Petaforce ${ }^{\circledR}$ au Canada). Schweiz Zschr GanzheitsMedizin 1995; 7: 365-370

13 Evers S. Pestwurz in der Behandlung der Migräne - Eine Übersicht. Nervenheilkunde 2009; 28: 548-552

14 Ko W, Lei C, Lin Y, Chen C. Relaxant effects of petasins in isolated guinea pig trachea and their structure-activity relationships. Planta Med 2000; 66: 650-652

15 Thomet OA, Wiesmann UN, Blaser K, Simon HU. Differential inhibition of inflammatory effector functions by petasin, isopetasin and neopetasin in human eosinophils. Clin Exp Allergy 2001; 31: 1310-1320

16 Ärztegesellschaft Heilfasten und Ernährung e.V. ExpertInnengruppe der ÄGHE: Wilhelmi de Toledo F, Buchinger A, Burggrabe $\mathrm{H}$ et al. Leitlinien zur Fastentherapie. Forsch Komplementarmed Klass Naturheilkunde 2002; 9: 189-198

17 Wilhelmi de Toledo F, Buchinger A, Burggrabe $\mathrm{H}$ et al. Fasting therapy - an expert panel update of the 2002 consensus guidelines. Forsch Komplementmed 2013; 20: 434-443 